\title{
2-Absorbing Semiprimary Fuzzy Ideal of Commutative Rings
}

\author{
Deniz Sönmez ${ }^{1}$, Gürsel Yeşilot ${ }^{1}$, Serkan $_{\text {Onar }}{ }^{*}$ and Bayram Ali Ersoy ${ }^{1}$ \\ ${ }^{1}$ Department of Mathematics, Faculty of Science and Arts, Yildiz Technical University, Turkey \\ ${ }^{2}$ Management Information Systems, Gelişim University, Turkey \\ ${ }^{*}$ Corresponding author E-mail: serkan10ar@gmail.com
}

\section{Article Info}

Keywords: 2-absorbing primary fuzzy ideals, 2-absorbing semiprimary fuzzy ideals 2010 AMS: 03E72, 08A72

Received: 01 July 2019

Accepted: 27 September 2019

Available online: 26 December 2019

\begin{abstract}
In this work, we introduce the notion of 2-absorbing semiprimary fuzzy ideal which is a generalization of semiprimary fuzzy ideal. Let $R$ be a ring. Then the nonconstant fuzzy ideal $\mu$ is called a 2-absorbing semiprimary fuzzy ideal if $\sqrt{\mu}$ is a 2-absorbing fuzzy ideal of $R$. Furthermore, we give some fundamental results concerning these notions.
\end{abstract}

\section{Introduction}

Zadeh in 1965 introduced the fundamental concept of fuzzy set [1]. Focusing on the structure of ring, the paper of Liu [2], defining fuzzy ideals, initiated the investigation of rings by means of expanding the class of ideals with these fuzzy objects. Mukherjee and Sen have continued the study of fuzzy ideals by introducing the notion of prime fuzzy ideals [3]. Nowadays, fuzzy algebraic structures were developed and many interesting results were obtained. The concept of 2-absorbing ideals, which is a generalization of prime ideals [4] and 2-absorbing primary ideals, which is a generalization of primary ideals [5] were introduced. Although the prime fuzzy ideals and primary fuzzy ideals have been investigated $[3,6]$, the concept of 2-absorbing semiprimary fuzzy ideals have not been studied yet. In this study, we characterize the 2-absorbing semiprimary fuzzy ideals, some generalizations of 2-absorbing semiprimary fuzzy ideals and described some their properties. Recall from [4, 5] that a proper ideal $I$ of $R$ is called a 2-absorbing ideal if whenever $a, b, c \in R$ and $a b c \in I$ then either $a b \in I$ or $a c \in I$ or $b c \in I$ and a proper ideal $I$ of $R$ is called a 2-absorbing primary ideal if whenever $a, b, c \in R$ and $a b c \in I$ then either $a b \in I$ or $a c \in \sqrt{I}$ or $b c \in \sqrt{I}$. Recall also from [7] that a nonconstant fuzzy ideal $\mu$ of $R$ is called a 2-absorbing fuzzy ideal of $R$ if for any fuzzy points $x_{r}, y_{s}, z_{t}$ of $R, x_{r} y_{s} z_{t} \in \mu$ implies that either $x_{r} y_{s} \in \mu$ or $x_{r} z_{t} \in \mu$ or $y_{s} z_{t} \in \mu$ and a nonconstant fuzzy ideal $\mu$ of $R$ is called a 2-absorbing primary fuzzy ideal of $R$ if for any fuzzy points $x_{r}, y_{s}, z_{t}$ of $R, x_{r} y_{s} z_{t} \in \mu$ implies that either $x_{r} y_{s} \in \mu$ or $x_{r} z_{t} \in \sqrt{\mu}$ or $y_{s} z_{t} \in \sqrt{\mu}$. Based on these definitions, a nonconstant fuzzy ideal $\mu$ is called a 2-absorbing semiprimary fuzzy ideal if $\sqrt{\mu}$ is a 2-absorbing fuzzy ideal of $R$.

\section{Preliminaries}

We assume throughout that all rings are commutative with $1 \neq 0$. Unless stated otherwise $L=[0,1]$ stands for a complete lattice. $\mathbb{Z}$ denotes the ring of integers, $L(R)$ denotes the set of fuzzy sets of $R$ and $L I(R)$ denotes the set of fuzzy ideals of $R$. For $\mu, \xi \in L(R)$, we say $\mu \subseteq \xi$ if and only if $\mu(x) \leq \xi(x)$ for all $x \in R$. When $r \in L, x, y \in R$ we define $x_{r} \in L(R)$ as follows :

$$
x_{r}(y)=\left\{\begin{array}{cc}
x & \text { if } x=y \\
0 & \text { otherwise }
\end{array}\right.
$$

and $x_{r}$ is referred to as fuzzy point of $R$. Let $I$ be an ideal of $R$. Then

$$
\lambda_{I}=\left\{\begin{array}{cc}
1 & \text { if } x \in I \\
0 & \text { otherwise }
\end{array}\right.
$$

Definition 2.1. [2] A fuzzy subset $\mu$ of a ring $R$ is called a fuzzy ideal of $R$ if for all $x, y \in R$ the following conditions are satisfied :

Email addresses and ORCID numbers: dnzguel@ hotmail.com, https://orcid.org/0000-0002-7574-4245 (D. Sönmez), gyesilot@yildiz.edu.tr, https://orcid.org/0000-00025205-5842 (G. Yeşilot),serkan10ar@gmail.com, https://orcid.org/0000-0003-3084-7694 (S. Onar),ersoya@gmail.com, https://orcid.org/0000-0002-8307-9644 (B. A. Ersoy) 
- $\mu(x-y) \geq \mu(x) \wedge \mu(y), \forall x, y \in R$

- $\mu(x y) \geq \mu(x) \vee \mu(y), \forall x, y \in R$

Let $\mu$ be any fuzzy ideal of $R ; x, y \in R$, and 0 be the additive identity of $R$. Then it is easy to verify the following:

(i) $\mu(0) \geq \mu(x), \mu(x)=\mu(-x)$ and $\mu_{t} \subset \mu_{s}$ where $s, t \in \operatorname{Im}(\mu)$ and $t>s$.

(ii) If $\mu(0)=\mu(x-y)$, then $\mu(x)=\mu(y), \mu(x)=s$ iff $x \in \mu_{s}$ and $x \notin \mu_{t}, \forall t>s$.

Definition 2.2. [8] Let $\mu$ be any fuzzy ideal of $R$. The ideals $\mu_{t},(\mu(0) \geq t)$ are called level ideals of $\mu$.

Definition 2.3. [3] A fuzzy ideal $\mu$ of $R$ is called prime fuzzy ideal if for any two fuzzy points $x_{r}, y_{s}$ of $R, x_{r} y_{s} \in \mu$ implies either $x_{r} \in \mu$ or $y_{s} \in \mu$.

Definition 2.4. [6] Let $\mu$ be a fuzzy ideal of $R$. Then $\sqrt{\mu}$, called the radical of $\mu$, is defined by $\sqrt{\mu}(x)=\underset{n \geq 1}{\vee} \mu\left(x^{n}\right)$.

Definition 2.5. [6] A fuzzy ideal $\mu$ of $R$ is called primary fuzzy ideal if for any two fuzzy points $x_{r}, y_{s}$ of $R, x_{r} y_{s} \in \mu$ implies either $x_{r} \in \mu$ or $y_{s} \in \sqrt{\mu}$.

Theorem 2.6. [6] Let $\mu$ be fuzzy ideal of a ring $R$. Then $\sqrt{\mu}$ is a fuzzy ideal of $R$.

Definition 2.7. [3] Let $R$ be a ring. Then a nonconstant fuzzy ideal $\mu$ is said to be weakly completely prime fuzzy ideal iff for $x, y \in R$, $\mu(x y)=\max \{\mu(x), \mu(y)\}$.

Theorem 2.8. [9] Let $f: R \rightarrow S$ be a ring homomorphism and let $\mu$ be a fuzzy ideal of $R$ such that $\mu$ is constant on Kerf and $\xi$ be a fuzzy ideal of $S$. Then,

- $\sqrt{f(\mu)}=f(\sqrt{\mu})$,

- $\sqrt{f^{-1}(\xi)}=f^{-1}(\sqrt{\xi})$.

Definition 2.9. [4] A nonzero proper ideal I of a commutative ring $R$ with $1 \neq 0$ is called a 2-absorbing ideal if whenever a,b,c $\in R$ with $a b c \in I$, then either $a b \in I$ or $a c \in I$ or $b c \in I$.

Definition 2.10. [5] A proper ideal I of $R$ is called a 2-absorbing primary ideal of $R$ if whenever $a, b, c \in R$ with abc $\in I$, then either $a b \in I$ or $a c \in \sqrt{I}$ or $b c \in \sqrt{I}$.

Definition 2.11. [10] A proper ideal $I$ of $R$ is called a 2-absorbing quasi primary ideal of $R$ if whenever a,b, $c \in R$ with abc $\in I$, then either $a b \in \sqrt{I}$ or $a c \in \sqrt{I}$ or $b c \in \sqrt{I}$.

Theorem 2.12. [5] If I is a 2-absorbing primary ideal of $R$, then $\sqrt{I}$ is a 2-absorbing ideal of $R$.

Definition 2.13. [11] An element $1>\alpha \in L$ is called a 2-absorbing element if for any $x, y, z \in L, x \wedge y \wedge z<\alpha$ implies either $x \wedge y<\alpha$ or $x \wedge z<\alpha$ or $y \wedge z<\alpha$.

Lemma 2.14. [9] Let $\mu$ be a fuzzy ideal of $R$. Then for any positive integer $n, \sqrt{\mu^{n}}=\sqrt{\mu}$.

Lemma 2.15. [9] Let $\mu$ and $\lambda$ be fuzzy ideals of $R$. If $\mu \subseteq \lambda$ then $\sqrt{\mu} \subseteq \sqrt{\lambda}$.

Theorem 2.16. [9] If $\mu$ and $\xi$ are two fuzzy ideals of $R$, then $\sqrt{\mu \cap \xi}=\sqrt{\mu} \cap \sqrt{\xi}=\sqrt{\mu \xi}$

Theorem 2.17. [7] $f: R \rightarrow S$ be a ring homomorphism. If $\xi$ is a 2-absorbing fuzzy ideal of $S$ then $f^{-1}(\xi)$ is a 2-absorbing fuzzy ideal of $R$.

Theorem 2.18. [7] Let $f: R \rightarrow S$ be a surjective ring homomorphism. If $\mu$ is a 2-absorbing fuzzy ideal of $R$ which is constant on Kerf then $f(\mu)$ is a 2-absorbing fuzzy ideal of $S$.

\section{2-absorbing semiprimary fuzzy ideals}

Before we investigate 2-absorbing semiprimary fuzzy ideals, we will give the characterization of cartesian product of some fuzzy ideals which will be used in next parts.

Definition 3.1. Let $\mu$ and $\alpha$ be two fuzzy ideals of $R$. The cartesian product of $\mu$ and $\alpha$ is defined by $\mu \times \alpha$ such that $(\mu \times \alpha)(x, y)=$ $\mu(x) \wedge \alpha(y)$ [12]. In addition to this definition, if $\left(x_{r}, y_{s}\right) \in \mu \times \alpha$ for any fuzzy points $x_{r}, y_{s}$ of $R$ then $x_{r} \in \mu$ and $y_{s} \in \alpha$ so $r \wedge s \leq$ $\mu \times \alpha(x, y)=\mu(x) \wedge \alpha(y)$.

Recall that if $\mu$ and $\alpha$ are fuzzy ideals of $R$ then $\mu \times \alpha$ is a fuzzy ideal of $R \times R$.

Lemma 3.2. Let $\mu$ and $\alpha$ be two fuzzy ideals of $R$. Then $\sqrt{\mu \times \alpha}=\sqrt{\mu} \times \sqrt{\alpha}$

Proof. $\sqrt{\mu \times \alpha}(x, y)=\bigvee_{n \geq 1}\left\{(\mu \times \alpha)\left(x^{n}, y^{n}\right)\right\}=\bigvee_{n \geq 1}\left\{\mu\left(x^{n}\right) \wedge \alpha\left(y^{n}\right)\right\}$

$=\bigvee_{n \geq 1}\left\{\mu\left(x^{n}\right)\right\} \wedge \bigvee_{n \geq 1}\left\{\alpha\left(y^{n}\right)\right\}=\sqrt{\mu}(x) \wedge \sqrt{\alpha}(y)=\sqrt{\mu} \times \sqrt{\alpha}(x, y)$

Lemma 3.3. Let $R=R_{1} \times R_{2}$, where $R_{1}$ and $R_{2}$ are rings and $\mu$ be a nonconstant fuzzy ideal of $R$. If $\mu$ is a prime fuzzy ideal then either $\mu=\mu_{1} \times \lambda_{R_{2}}$ for some prime fuzzy ideal $\mu_{1}$ of $R_{1}$ or $\mu=\lambda_{R_{1}} \times \mu_{2}$ for some prime fuzzy ideal $\mu_{2}$ of $R_{2}$.

Proof. Assume that $\mu$ be a prime fuzzy ideal of $R$. Then there exist $\alpha$ and $\beta$ fuzzy ideals of $R_{1}, R_{2}$ respectively such that $\mu=\alpha \times \beta$. Then for any fuzzy points $x_{r}, y_{s}$ of $R\left(x_{r}, y_{s}\right)=\left(x_{r}, 1_{1}\right)\left(1_{1}, y_{s}\right) \in \mu=\alpha \times \beta$. So $\left(x_{r}, 1_{1}\right) \in \alpha \times \beta$ or $\left(1_{1}, y_{s}\right) \in \alpha \times \beta$ since $\mu$ is a prime fuzzy ideal. Thus we conclude that $\beta=\lambda_{R_{2}}$ and $\alpha$ is a prime fuzzy ideal or $\alpha=\lambda_{R_{1}}$ and $\beta$ is a prime fuzzy ideal. 
Theorem 3.4. Let $R=R_{1} \times R_{2}$, where $R_{1}$ and $R_{2}$ are commutative rings with nonzero identity. Let $\mu$ be a nonconstant fuzzy ideal of $R$. Then the following statements are equivalent:

(1) $\mu$ is a semiprimary fuzzy ideal of $R$.

(2) Either $\mu=\mu_{1} \times \lambda_{R_{2}}$ for some semiprimary fuzzy ideal $\mu_{1}$ of $R_{1}$ or $\mu=\lambda_{R_{1}} \times \mu_{2}$ for some semiprimary fuzzy ideal $\mu_{2}$ of $R_{2}$.

Proof. (1) $\Rightarrow$ (2) Assume that $\mu$ is a semiprimary fuzzy ideal of $R$. Then $\mu=\mu_{1} \times \mu_{2}$ for some fuzzy ideal $\mu_{1}$ of $R_{1}$ and some fuzzy ideal $\mu_{2}$ of $R_{2}$. Since $\sqrt{\mu}=\sqrt{\mu_{1}} \times \sqrt{\mu_{2}}$ is a prime fuzzy ideal then by the previous lemma either $\sqrt{\mu_{1}}=\lambda_{R_{1}}$ so $\mu_{1}=\lambda_{R_{1}}$ and $\sqrt{\mu_{2}}$ is a prime fuzzy ideal or $\sqrt{\mu_{2}}=\lambda_{R_{2}}$ so $\mu_{2}=\lambda_{R_{2}}$ and $\sqrt{\mu_{1}}$ is a prime fuzzy ideal. Hence $\mu=\mu_{1} \times \lambda_{R_{2}}$ for some semiprimary fuzzy ideal $\mu_{1}$ or $\mu=\lambda_{R_{1}} \times \mu_{2}$ for some semiprimary fuzzy ideal $\mu_{2}$.

(2) $\Rightarrow$ (1) It is clear that since $\sqrt{\mu}=\sqrt{\mu_{1} \times \lambda_{R_{2}}}=\sqrt{\mu_{1}} \times \lambda_{R_{2}}$ is a prime fuzzy ideal of $R$ if $\mu_{1}$ is any semiprimary fuzzy ideal of $R_{1}$.

Theorem 3.5. Let $\mu$ be a fuzzy ideal of $R$. If $\mu$ is a 2-absorbing then $\mu \times \lambda_{R}\left(\lambda_{R} \times \mu\right)$ is a 2-absorbing fuzzy ideal of $R \times R$.

Proof. Assume that $\left(x_{r}, a_{k}\right)\left(y_{s}, b_{p}\right)\left(z_{t}, c_{h}\right) \in \mu \times \lambda_{R}$ for any fuzzy points $x_{r}, y_{s}, z_{t}, a_{k}, b_{p}, c_{h}$ of $R$. Then $\left(x_{r} y_{s} z_{t}, a_{k} b_{p} c_{h}\right) \in \mu \times \lambda_{R}$ so $x_{r} y_{s} z_{t} \in \mu$ and $a_{k} b_{p} c_{h} \in \lambda_{R}$. Since $\mu$ is a 2-absorbing fuzzy ideal then $x_{r} y_{s} \in \mu$ or $x_{r} z_{t} \in \mu$ or $y_{s} z_{t} \in \mu$. Thus we get that $\left(x_{r}, a_{k}\right)\left(y_{s}, b_{p}\right) \in \mu \times \lambda_{R}$ or $\left(x_{r}, a_{k}\right)\left(z_{t}, c_{h}\right) \in \mu \times \lambda_{R}$ or $\left(y_{s}, b_{p}\right)\left(z_{t}, c_{h}\right) \in \mu \times \lambda_{R}$. Hence $\mu \times \lambda_{R}$ is a 2-absorbing fuzzy ideal.

By the similar way it can be seen that $\lambda_{R} \times \mu$ is a 2-absorbing fuzzy ideal of $R \times R$.

Definition 3.6. Let $R$ be a ring. Then the nonconstant fuzzy ideal $\mu$ is said to be a 2-absorbing semiprimary fuzzy ideal if $\sqrt{\mu}$ is a 2-absorbing fuzzy ideal of $R$.

Example 3.7. (1) Every prime fuzzy ideal is a 2-absorbing semiprimary fuzzy ideal.

(2) Every primary fuzzy ideal is a 2-absorbing semiprimary fuzzy ideal.

(3) Every semiprimary fuzzy ideal is a 2-absorbing semiprimary fuzzy ideal.

Proposition 3.8. Let $\mu$ be a nonconstant fuzzy ideal of $R$. Then the following assertions are equivalent.

(i) $\mu$ is a 2-absorbing semiprimary fuzzy ideal.

(ii) If $x_{r} y_{s} z_{t} \in \mu$ for any fuzzy points $x_{r}, y_{s}, z_{t}$ of $R$ then $x_{r} y_{s} \in \sqrt{\mu}$ or $x_{r} z_{t} \in \sqrt{\mu}$ or $y_{s} z_{t} \in \sqrt{\mu}$.

Corollary 3.9. If $\mu$ is 2-absorbing primary fuzzy ideal then $\mu$ is 2-absorbing semiprimary fuzzy ideal. But, as indicated in the following example, the converse of Corollary 3.9 is not true.

Example 3.10. Let $R=\mathbb{Z}$, the ring of integers. Define the fuzzy ideal $\mu$ of $Z$ by

$$
\mu(x)=\left\{\begin{array}{cc}
1 & x \in 36 Z \\
1 / 2 & x \in 6 Z-36 Z \\
0 & \text { otherwise }
\end{array}\right.
$$

Since $2_{1} 3_{1} \notin \mu, 2_{1} 1_{\frac{1}{2}} \notin \sqrt{\mu}$ and $3_{1} 1_{\frac{1}{2}} \notin \sqrt{\mu}$ while $2_{1} 3_{1} 1_{\frac{1}{2}} \in \mu$, then $\mu$ is not 2-absorbing primary fuzzy ideal. However, it is easy to see that $\mu$ is 2-absorbing semiprimary fuzzy ideal, since $\sqrt{\mu}=\lambda_{6 Z}$ where it is a 2-absorbing fuzzy ideal of $Z$.

Remark 3.11. In Example 2.7 [5], it is proved that a 2-absorbing semiprimary ideal is not necessarily a 2-absorbing primary ideal. In the following theorem we show under what conditions a 2-absorbing semiprimary (fuzzy) ideal is a 2-absorbing primary (fuzzy) ideal. Note that if $\mu$ is a semiprime fuzzy ideal of $R$, then we have $\sqrt{\mu}=\mu$.

Theorem 3.12. Let $R$ be a ring. Then the following statements hold:

(1) Let $\mu$ be a semiprime fuzzy ideal of $R$. Then $\mu$ is a 2-absorbing primary fuzzy ideal if and only if it is 2-absorbing semiprimary fuzzy ideal.

(2) Let I be a semiprime ideal of R. Then I is a 2-absorbing primary ideal if and only if it is 2-absorbing semiprimary ideal.

Proof. (1) We show that only sufficient conditions. Let $\mu$ be semiprime fuzzy ideal. If $\mu$ is 2-absorbing semiprimary fuzzy ideal and $x_{r} y_{s} z_{t} \in \mu$ for any $x_{r}, y_{s}, z_{t}$ fuzzy points of $R$, then $x_{r} y_{s} \in \sqrt{\mu}$ or $x_{r} z_{t} \in \sqrt{\mu}$ or $y_{s} z_{t} \in \sqrt{\mu}$. Since $\mu$ is semiprime fuzzy ideal then $\sqrt{\mu}=\mu$ so $x_{r} y_{s} \in \mu=\sqrt{\mu}$ or $x_{r} z_{t} \in \sqrt{\mu}$ or $y_{s} z_{t} \in \sqrt{\mu}$. Hence we get that $\mu$ is a 2-absorbing primary fuzzy ideal of $R$.

(2) We omit the proof since it is clear by (1).

Theorem 3.13. Let $\mu$ be a fuzzy ideal of $R$. If $\mu$ is a 2-absorbing semiprimary then $\mu_{t}$ is a 2-absorbing semiprimary ideal of $R$ for any $t \in[0, \mu(0)]$.

Proof. If $\mu$ is a 2-absorbing semiprimary then $\sqrt{\mu}$ is a 2-absorbing fuzzy ideal of $R$. By [7, Lemma 3.3], $\sqrt{\mu}_{t}=\sqrt{\mu_{t}}$ is also 2-absorbing ideal. Hence $\mu_{t}$ is 2-absorbing semiprimary ideal of $R$.

Theorem 3.14. Let $\mu_{1}$ be $\xi_{1}$-semiprimary fuzzy ideal of $R$ and $\mu_{2}$ be $\xi_{2}$-semiprimary fuzzy ideal of $R$. Then the following statements hold. (i) $\mu_{1} \mu_{2}$ is a 2-absorbing semiprimary fuzzy ideal of $R$.

(ii) $\mu_{1} \cap \mu_{2}$ is a 2-absorbing semiprimary fuzzy ideal of $R$.

Proof. Since $\sqrt{\mu_{1}}=\xi_{1}$ and $\sqrt{\mu_{2}}=\xi_{2}$ are prime fuzzy ideals then $\sqrt{\mu_{1} \mu_{2}}=\sqrt{\mu_{1} \cap \mu_{2}}=\sqrt{\mu_{1}} \cap \sqrt{\mu_{2}}$ is 2-absorbing fuzzy ideal of $R$. Hence $\mu_{1} \cap \mu_{2}$ and $\mu_{1} \mu_{2}$ are 2-absorbing semiprimary fuzzy ideal.

Theorem 3.15. Let $\mu$ be a nonconstant fuzzy ideal. If $\mu_{*}=\{x \in R: \mu(x)>0\}$ is a 2-absorbing semiprimary ideal of $R$ where $\mu(0)=1$ and $|\operatorname{Im} \mu|=2$ then $\mu$ is a 2-absorbing semiprimary fuzzy ideal of $R$. 
Proof. Assume that $\mu(0)=1, \operatorname{Im} \mu=\{1, \alpha\}$ and $\mu_{*}$ is a 2-absorbing semiprimary ideal.

Let $x_{r} y_{s} z_{t} \in \mu$ but $x_{r} y_{s} \notin \sqrt{\mu}, y_{s} z_{t} \notin \sqrt{\mu}$ and $x_{r} z_{t} \notin \sqrt{\mu}$. Then $r \wedge s \wedge t \leq \mu(x y z)$ and $r \wedge s>\sqrt{\mu}(x y), s \wedge t>\sqrt{\mu}(y z), r \wedge t>\sqrt{\mu}(x z)$. Thus for all $n \in \mathbb{Z}^{+}, r \wedge s>\mu\left(x^{n} y^{n}\right), s \wedge t>\mu\left(y^{n} z^{n}\right)$ and $r \wedge t>\mu\left(x^{n} z^{n}\right)$. By our assumption we get that $\mu\left(x^{n} y^{n}\right)=\mu\left(y^{n} z^{n}\right)=\mu\left(x^{n} z^{n}\right)=\alpha$ so $x y, y z, x z \notin \mu_{*}$. However, $\alpha<r \wedge s \wedge t \leq \mu(x y z)=1$ so $x y, y z, x z \notin \sqrt{\mu_{*}}$ and $x y z \in \mu_{*}$. But this contradict that $\mu_{*}$ is 2 -absorbing semiprimary ideal. Hence $\mu$ is a 2-absorbing semiprimary fuzzy ideal.

Theorem 3.16. Let I be a 2-absorbing quasi primary ideal of $R$ and $\alpha \in[0,1)$ be any arbitrary. If $\mu$ is the fuzzy ideal of $R$ defined by

$$
\mu(x)= \begin{cases}1 & x \in I \\ \alpha & x \notin I\end{cases}
$$

for all $x \in R$, then $\mu$ is a 2-absorbing semiprimary fuzzy ideal of $R$.

Proof. Let $I$ be a 2-absorbing primary ideal of $R$. Assume that $x_{r} y_{s} z_{t} \in \mu$ but $x_{r} y_{s} \notin \sqrt{\mu}$ and $x_{r} z_{t} \notin \sqrt{\mu}$ and $y_{s} z_{t} \notin \sqrt{\mu}$ for any $x, y, z \in R$. Then $\mu\left((x y)^{n}\right) \leq \sqrt{\mu}(x y)<r \wedge s$ and $\mu\left((y z)^{n}\right) \leq \sqrt{\mu}(y z)<s \wedge t$ and $\mu\left((x z)^{n}\right) \leq \sqrt{\mu}(x z)<r \wedge t$ for all $n \geq 1$. In this case $\mu\left((x y)^{n}\right)=\alpha$ and $(x y)^{n} \notin I$ so $x y \notin \sqrt{I}, \mu\left((y z)^{n}\right)=\alpha$ and $(y z)^{n} \notin I$ so $y z \notin \sqrt{I}, \mu\left((x z)^{n}\right)=\alpha$ and $(x z)^{n} \notin I$ so $x z \notin \sqrt{I}$. Since $I$ is 2-absorbing semiprimary ideal of $R$ then we get $x y z \notin I$ and so $\mu(x y z)=\alpha$. By our assumption we get $(x y z)_{r \wedge s \wedge t}=x_{r} y_{s} z_{t} \in \mu$ and $r \wedge s \wedge t \leq \mu(x y z)=\alpha$. Thus $\alpha<r \wedge s, \alpha<s \wedge t$ and $\alpha<r \wedge t$ so $\alpha<r \wedge s \wedge t$, which is a contradiction. Hence $\mu$ is a 2-absorbing semiprimary fuzzy ideal of $R$.

Theorem 3.17. Let $f: R \rightarrow S$ be a ring homomorphism. If $\xi$ is a 2-absorbing semiprimary fuzzy ideal of $S$ then $f^{-1}(\xi)$ is a 2-absorbing semiprimary fuzzy ideal of $R$.

Proof. Let $\xi$ be a 2-absorbing semiprimary fuzzy ideal of S. We show that $\sqrt{f^{-1}(\xi)}$ is a 2-absorbing fuzzy ideal of $R$. Since $\sqrt{f^{-1}(\xi)}=$ $f^{-1}(\sqrt{\xi})$ and $\sqrt{\xi}$ is a 2-absorbing fuzzy ideal then the inverse image of $\sqrt{\xi}$ is also 2-absorbing fuzzy ideal by [7, Theorem 31]. Hence $f^{-1}(\xi)$ is a 2-absorbing semiprimary fuzzy ideal of $R$.

Theorem 3.18. Let $f: R \rightarrow S$ be a surjective ring homomorphism. If $\mu$ is a 2-absorbing semiprimary fuzzy ideal of $R$ which is constant on Kerf then $f(\mu)$ is a 2-absorbing semiprimary fuzzy ideal of $S$.

Proof. Assume that $\mu$ is a 2-absorbing semiprimary fuzzy ideal of $R$ which is constant on $\operatorname{Kerf}$. Then $\sqrt{\mu}$ is a 2-absorbing fuzzy ideal of $R$ such that $\sqrt{\mu}$ is also constant on $\operatorname{Kerf}$. By [7, Theorem 32], $f(\sqrt{\mu})=\sqrt{f(\mu)}$ is a 2-absorbing fuzzy ideal of $S$.

Theorem 3.19. If $f$ is a homomorphism from a ring $R$ onto a ring $S$, then the mapping $\mu \rightarrow f(\mu)$ defines a one-to-one correspondence between the set of all 2-absorbing semiprimary fuzzy ideals of $R$ which is constant on Kerf and the set of all 2-absorbing semiprimary fuzzy ideals of $S$.

Definition 3.20. Let $\mu$ be a 2-absorbing semiprimary fuzzy ideal of $R$. Then $\gamma=\sqrt{\mu}$ is a 2-absorbing fuzzy ideal. We say that $\mu$ is a $\gamma$-2-absorbing semiprimary fuzzy ideal of $R$.

Theorem 3.21. Let $\mu_{1}, \mu_{2}, \ldots, \mu_{n}$ be $\gamma$-2-absorbing semiprimary fuzzy ideals of $R$ for some 2-absorbing fuzzy ideal $\gamma$ of $R$. Then $\mu=\bigcap_{i=1}^{n} \mu_{i}$ is a $\gamma$-2-absorbing semiprimary fuzzy ideal of $R$.

Proof. Let $\mu_{i}, i \in\{1,2, \ldots, n\}$ be $\gamma$-2-absorbing semiprimary fuzzy ideals of $R$. Then $\sqrt{\bigcap_{i=1}^{n} \mu_{i}}=\bigcap_{i=1}^{n} \sqrt{\mu_{i}}=\gamma=\sqrt{\mu}$ is a 2-absorbing fuzzy ideal. Hence $\mu$ is a $\gamma$-2-absorbing primary fuzzy ideal of $R$.

Theorem 3.22. Let $R_{1}$ and $R_{2}$ be commutative rings with nonzero identity and $\mu$ be a nonconstant fuzzy ideal of $R_{1}$ (of $R_{2}$ ). If $\mu$ is a 2-absorbing semiprimary fuzzy ideal of $R_{1}$ (of $\left.R_{2}\right)$ then $\mu \times \lambda_{R_{2}}\left(\lambda_{R_{1}} \times \mu\right)$ is a 2-absorbing semiprimary fuzzy ideal of $R_{1} \times R_{2}$.

Proof. Assume that $\mu$ is a 2-absorbing semiprimary fuzzy ideal. Since $\sqrt{\mu}$ is a 2-absorbing fuzzy ideal then $\sqrt{\mu \times \lambda_{R_{2}}}=\sqrt{\mu} \times \lambda_{R_{2}}$ is a 2-absorbing fuzzy ideal of $R_{1} \times R_{2}$. Hence we get $\mu \times \lambda_{R_{2}}$ is a 2 -absorbing semiprimary fuzzy ideal of $R_{1} \times R_{2}$.

Corollary 3.23. Let $R=R_{1} \times R_{2}$ where $R_{1}$ and $R_{2}$ be two rings and $\mu$ be a nonconstant fuzzy ideal of $R$. Then the following statements are equivalent: (1) $\mu$ is a 2-absorbing semiprimary fuzzy ideal of $R$. (2) Either $\mu=\mu_{1} \times \lambda_{R_{2}}$ for some 2-absorbing semiprimary fuzzy ideal $\mu_{1}$ of $R_{1}$, or $\mu=\lambda_{R_{1}} \times \mu_{2}$ for some 2-absorbing semiprimary fuzzy ideal $\mu_{2}$ of $R_{2}$, or $\mu=\mu_{1} \times \mu_{2}$ for some 2-absorbing semiprimary fuzzy ideal of $R_{2}$.

\section{Conclusion}

In this paper, we have characterized 2-absorbing semiprimary fuzzy ideals of a ring. Also the notions of 2-absorbing and 2-absorbing primary fuzzy ideals and their properties are proposed. Furthermore, the relationship between 2-absorbing semiprimary fuzzy ideals and 2-absorbing semiprimary ideals. Finally, we have examined that the properties of cartesian product of 2-absorbing semiprimary fuzzy ideals. To extend this study, one could study other algebraic structures and do some further study on the properties them.

\section{Acknowledgement}

This work was supported by Research Fund of the Yildiz Technical University. Project Number: 2015-01-03-DOP03. 


\section{References}

[1] L. A. Zadeh, Fuzzy sets, Inform. Control, 8 (1965), 338-353.

[2] W. J. Liu, Fuzzy invariant subgroups and fuzzy ideals, Fuzzy Sets Syst., 8 (1982), 133-139.

[3] T. K. Mukherjee, M.K. Sen, Prime fuzzy ideals in rings, Fuzzy Sets Syst. 32 (1989), 337-341.

[4] A. Badawi, On 2-absorbing ideals of commutative rings, Bull. Austral. Math. Soc., 75(3) (2007), 417-429.

[5] A. Badawi, U. Tekir, E. Yetkin, On 2-absorbing primary ideals in commutative rings, Bull. Austral. Math. Soc., 51(4) (2014), 1163-1173.

[6] T. K. Mukherjee, M. K. Sen, Primary fuzzy ideals and radical of fuzzy ideals, Fuzzy Sets Syst., 56 (1993), 97-101.

[7] D. Sönmez, G. Yeşilot, S. Onar, B. A. Ersoy, B. Davvaz, On 2-absorbing primary fuzzy ideals of commutative rings, Math. Probl. Eng., (2017), doi:

[8] V. N. Dixit, R. Kumar, N. Ajmal. Fuzzy ideals and fuzzy prime ideals of a ring, Fuzzy Sets Syst., 44 (1991), 127-138.

[9] L. I. Sidky, S. A. Khatab, Nil radical of fuzzy ideal, Fuzzy Sets Syst., 47 (1992), 117-120.

[10] S. Koc, R. N. Uregen, U. Tekir. On 2-absorbing quasi primary submodules, Filomat, 31 (2017), 2943-2950.

[11] F. Callialp, E. Yetkin, U. Tekir, On 2-absorbing primary and weakly 2-absorbing primary elements in multiplicative lattices, Ital. J. Pure Appl. Math., 34 (2015), 263-276.

[12] B. A. Ersoy, A generalization of cartesian product of fuzzy subgroups and ideals, J. Appl. Sci., 3 (2003), $100-102$. 